OPEN ACCESS

Edited by:

Pei Xu,

China Jiliang University, China

Reviewed by:

Xiang Lin,

Huazhong Agricultural

University, China

Haibin Wang,

Nanjing Agricultural University, China

${ }^{*}$ Correspondence:

Xiaole Wang

35337650@qq.com

Zhongjian Liu

zjliu@fafu.edu.cn

${ }^{\dagger}$ Present address: Xiaole Wang

Ningbo Academy of Agricultural

Sciences, Ningbo, China

Specialty section

This article was submitted to

Crop and Product Physiology,

a section of the journal

Frontiers in Plant Science

Received: 07 July 2021 Accepted: 07 September 2021

Published: 12 October 2021

Citation:

Zhou Z, Ying Z, Wu Z, Yang Y, Fu S, Xu W, Yao L, Zeng A, Huang J, Lan S, Wang $X$ and Liu $Z$ (2021) Anthocyanin

Genes Involved in the Flower Coloration Mechanisms of Cymbidium kanran. Front. Plant Sci. 12:737815. doi: 10.3389/fpls.2021.737815

\section{Anthocyanin Genes Involved in the Flower Coloration Mechanisms of Cymbidium kanran}

\author{
Zhuang Zhou ${ }^{1}$, Zhen Ying ${ }^{1}$, Zhigang Wu ${ }^{1}$, Yanping Yang ${ }^{1}$, Shuangbin $F u^{1}$, Wan $\mathrm{Xu}^{1}$, \\ Lijuan Yao ${ }^{1}$, Aiping Zeng ${ }^{1}$, Jian Huang ${ }^{1}$, Siren Lan ${ }^{2}$, Xiaole Wang ${ }^{1{ }^{* t}}$ and Zhongjian Liu ${ }^{1 *}$ \\ ${ }^{1}$ Zhejiang Institute of Subtropical Crops, Zhejiang Academy of Agricultural Sciences, Wenzhou, China, ${ }^{2}$ Key Laboratory of \\ National Forestry and Grassland Administration for Orchid Conservation and Utilization at College of Landscape Architecture, \\ Fujian Agriculture and Forestry University, Fuzhou, China
}

The Orchidaceae, otherwise known as orchids, is one of the largest plant families and is renowned for its spectacular flowers and ecological adaptations. Various polymorphisms of orchid flower colour can attract pollinators and be recognised as valuable horticultural ornamentals. As one of the longest historic cultured orchids, Cymbidium kanran has been domesticated for more than 2,500 years and is an ideal species to study coloration mechanisms because of plentiful variations in floral coloration and abundant traditional varieties. In this study, we used two distinct colour-type flowers of $C$. kanran as experimental materials to elucidate the mechanism of flower coloration. High-performance liquid chromatography (HPLC) analysis revealed that anthocyanins in purple-red-type flowers include three types of anthocyanidin aglycones, peonidin, malvidin, and cyanidin, whereas anthocyanins are lacking in white-type flowers. Through comparative transcriptome sequencing, 102 candidate genes were identified as putative homologues of colour-related genes. Based on comprehensive correlation analysis between colour-related compounds and gene expression profiles, four candidates from 102 captured genes showed a positive correlation with anthocyanidin biosynthesis. Furthermore, transient expression of CkCHS-1, CkDFR, and CKANS by particle bombardment confirmed that recovery of their expression completed the anthocyanin pathway and produced anthocyanin compounds in white-type flowers. Collectively, this study provided a comprehensive transcriptomic dataset for Cymbidium, which significantly facilitate our understanding of the molecular mechanisms of regulating floral pigment accumulation in orchids.

Keywords: orchids, Cymbidium kanran, anthocyanins, CkCHS-1, CkDFR, CkANS, flower coloration

\section{INTRODUCTION}

Orchids are important ornamental plants characterised by their fantastic floral morphology and high levels of corolla-colour polymorphism and variability, which have played an important role in pollination and have excited biologists since Darwin (Gigord et al., 2001; Aguiar et al., 2020; Basist et al., 2021). As an important member of orchids, Cymbidium species are probably the earliest orchid, having been cultivated since before the time of Confucius (551-479 BC) for traditional use in worship and ornamentation (Chen and Tang, 1982; Du Puy et al., 2007). Currently, members of 
Cymbidium are still very prevalent and dominate the world floriculture markets (Singh et al., 2019). Although the mechanism of genes encoding enzymes for flower coloration in orchids has been studied in some species (Mudalige-Jayawickrama et al., 2005; Han et al., 2006; Hieber et al., 2006; Chen et al., 2011; Hsiao et al., 2011; Liu et al., 2012; Yu et al., 2018), as one of the longest historic floricultural plants, Cymbidium kanran attracts wide attention. However, its coloration mechanism remains unclear.

Anthocyanins, as a series of secondary metabolites, are water-soluble pigments that contribute colours from orange, pink, red, magenta, purple, blue, cyan to "black" (Hatier and Gould, 2007; Tanaka et al., 2008; Petroni and Tonelli, 2011; Moreau et al., 2012; Li et al., 2014). These pigments are an ideal model for genetics, molecular biology, and cell biology (Passeri et al., 2016). To date, hundreds of anthocyanins have been extensively studied and characterised, which were originally based on six common types of anthocyanidins (chromophores of anthocyanins), namely, petunidin, pelargonidin, delphinidin, cyanidin, malvidin, and peonidin. Accordingly, the synthetic route of anthocyanins and important related genes are wellstudied and characterised in some model and non-model plants. Anthocyanins are derived from flavonoid biosynthetic pathways, and their biosynthesis has been divided into three stages (Grotewold, 2006). The first stage is phenylalanine and phenylpropanoid metabolism, which is shared among other secondary metabolisms. The second stage, flavonoid metabolism, is the crux of anthocyanin biosynthesis. Chalcone synthase (CHS) is the first key enzyme to produce chalcones, the precursor for all classes of flavonoids. Subsequently, an enzymatic reaction of chalcones to naringenins is catalysed by chalcone isomerase $(\mathrm{CHI})$, which is followed by a conversion to dihydrokaempferol (DHK) by flavanone 3-hydroxylase $(\mathrm{F} 3 \mathrm{H})$. The last step involves two participants, flavonoid 3'-hydroxylase (F3'H) and flavonoid 3', 5’hydroxylase (F3' 5'H), corresponding to the respective products dihydroquercetin (DHQ) and dihydromyricetin (DHM). The final stage is anthocyanin metabolism, including two rate-limiting steps catalysed by dihydroflavonol reductase (DFR) and anthocyanidin synthase (ANS). Three kinds of products, delphinidin, pelargonidin, and cyanidin, undergo several modifications, such as glycosylation or methylation, by UDP-glucoside: flavonoid 3-O-glucosyltransferase (3GT) and anthocyanin methyltransferase (AMT).

Transgenic technology, expression, and suppression of the level of the anthocyanin synthetic gene have been verified as feasible to study the accumulation mechanism of anthocyanin in many plants. With the expression of UFGT, the skin colours of the Vitis and Litchi were turned red or violet from green (Ramazzotti et al., 2008; Zhao et al., 2012). Overexpression of SmANS from Salvia miltiorrhiza enhanced anthocyanin accumulation in the petals of $S$. miltiorrhiza f. alba and resulted in purple-red colour from white colour (Li et al., 2019). Using RNA interference (RNAi) technology to suppress CHS, ANS, and $F 3^{\prime} 5^{\prime} H$, the petals of gentian plants exhibited pure white to pale-blue and magenta flowers accordingly (Nakatsuka et al., 2008). Consequently, using transgenic approaches to introduce exogenous anthocyanin genes into the targeted plant can obtain the desired coloration traits to produce new flower colour (Meyer et al., 1987; Katsumoto et al., 2007).

Cymbidium kanran is an ideal species to study coloration mechanisms because of numerous variations in floral coloration and abundant traditional varieties. In this study, we used purple-red flowers (with many purple-red pigments) and white flowers (greenish-white without purple-red pigments) as research materials (Figure 1). Through a combination of transcriptome sequencing, chemical, and molecular analysis, the mechanism of anthocyanin deficiency in white was preliminarily revealed. This dataset provided a novel resource to study and to understand the molecular mechanisms of coloration in C. kanran.

\section{MATERIALS AND METHODS}

\section{Plant Material and Freehand Sectioning}

White-type flowers and purple-red-type flowers of C. kanran were used as research materials in this study and cultivated in the greenhouse of Zhejiang Institute of Subtropical Crops in Wenzhou City, China $\left(120^{\circ} 37^{\prime} 53^{\prime \prime} \mathrm{E}, 28^{\circ} 0^{\prime} 8^{\prime \prime} \mathrm{N}\right)$. For the first one, it has white flowers (Figure 1A), for the second, it has purple-red flowers with purple-red sepals/petals and white lip with purple-red spots (Figure 1B). The buds were about $2,3 \mathrm{~cm}$, 1-2 days before anthesis, and opened flowers were used for RealTime PCR analysis. At the same time, about $3 \mathrm{~cm}$ length buds were selected for transcriptome sequencing and fully opened flowers were selected for freehand sectioning and pigment analysis. When harvested, all the materials were immediately frozen in liquid nitrogen (LN) and stored at $-80^{\circ} \mathrm{C}$ except the freehand sectioning samples. Petals or lips of fully opened flowers were perpendicularly cut quickly to their longitudinal axis by hand sectioning with a new sharp double-edged razor blade. The sections were immersed in water and observed under an optical microscope.

\section{RNA Extraction, Library Construction, and RNA-seq}

The total RNA of each sample was isolated using TRIzol Reagent (Invitrogen Life Technologies, Carlsbad, CA, USA). Then, the integrity and concentration were checked on a $1 \%$ agarose gel and a NanoDrop ND-1000 spectrophotometer (NanoDrop Technologies, Wilmington, DE, USA). The enriched mRNA was broken into short fragments, which were used to synthesise first- and second-strand cDNA. After that, the construction of the cDNA library was performed following the instructions of the manufacturer of the NEBNext Ultra RNA Library Prep Kit for Illumina (NEB, E7530, New England Biolabs, Ipswich, MA, USA) and NEBNext Multiplex Oligos for Illumina (NEB, E7500). Finally, the libraries of $C$. kanran buds were sequenced using an Illumina HiSeq ${ }^{\mathrm{TM}} 2500$ by Biomarker Biotechnology Corporation (Beijing, China).

\section{RNA-seq Data Assembly, Annotation, and Differential Expression Analysis}

Raw data obtained by RNA-seq were primarily processed through in-house Perl scripts. In this step, clean reads were acquired 

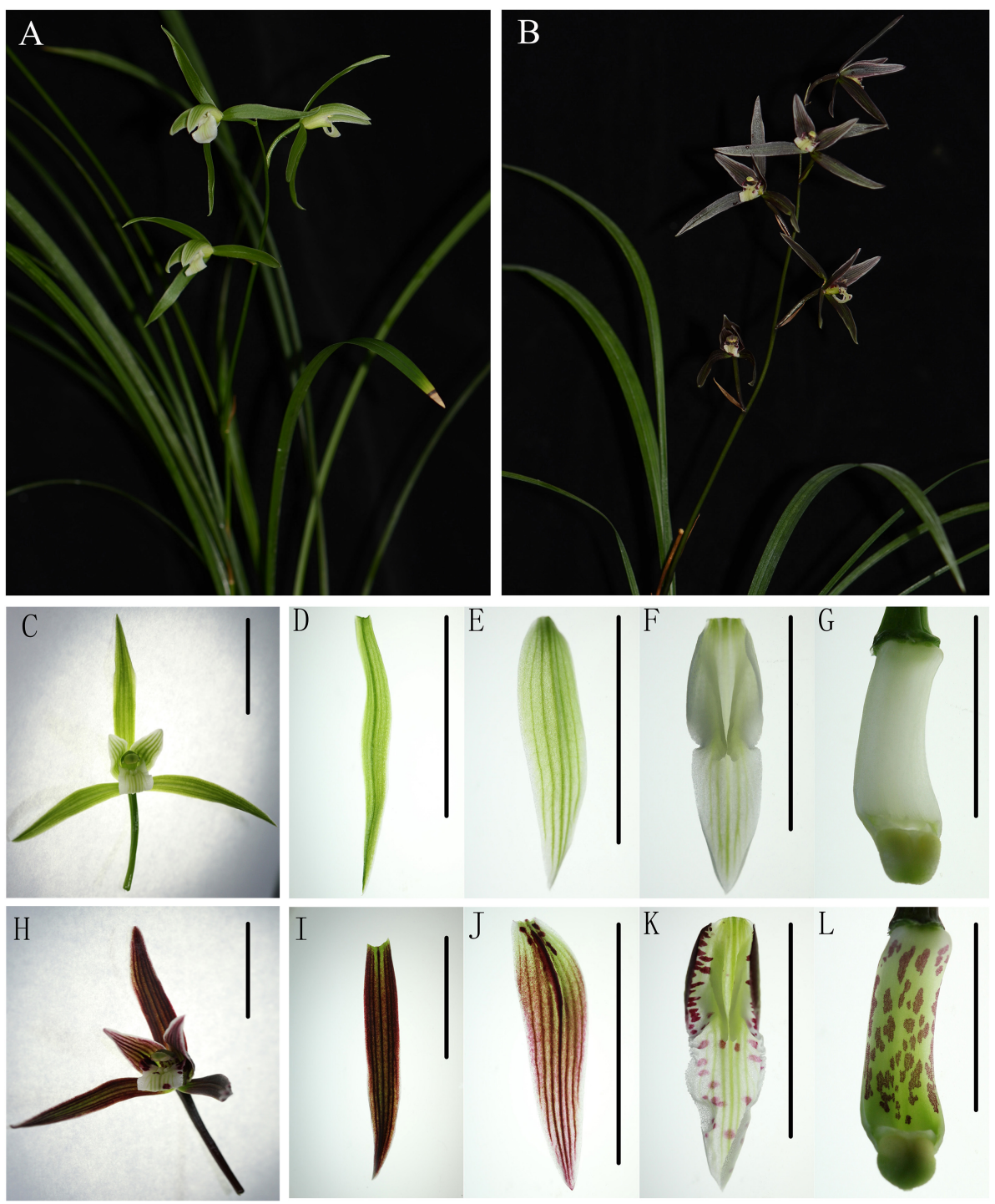

FIGURE 1 | The phenotype of purple-red and white Cymbidium kanran. (A) The plant of purple-red C. kanran. (B) The plant of white C. kanran. (C-G) Flower, sepal, petal, lip, and gynostemium of white C. kanran. (H-L) Flower, sepal, petal, lip, and gynostemium of purple-red C. kanran. Scale bar = $1 \mathrm{~cm}$.

by removing reads containing adapters, reads containing poly$\mathrm{N}$ and low-quality reads from raw data. In addition, the Q20, Q30, GC content, and sequence duplication levels of the clean data were calculated to evaluate the sequencing quality. All the subsequent analyses were based on clean data with high quality. Transcriptome assembly was accomplished based on the fq file using Trinity software (https:/github.com/ trinityrnaseq/trinityrnaseq/wiki) (Grabherr et al., 2011) with min_kmer_cov set to two by default and all other parameters set to default.

Gene function was annotated by BLASTX (Altschul et al., 1997) program (http://www.ncbi.nlm.nih.gov/) based on the following databases: $\mathrm{Nr}$ (NCBI non redundant protein sequences); Nt (NCBI non-redundant nucleotide sequences); Pfam (Protein family); KOG/COG (Clusters of Orthologous Groups of proteins); Swiss-Prot (a manually annotated and reviewed protein sequence database); KO (KEGG Orthologue database); and GO (Gene Ontology).

Gene expression levels were estimated using RSEM software (http://deweylab.github.io/RSEM/) (Li and Dewey, 2011) for each sample. Differential expression analysis of the two samples was performed using DESeq (http://www.bioconductor. org/packages/3.8/bioc/html/DESeq.html). The resulting $P$-values were adjusted with the Benjamini-Hochberg method to control the false discovery rate. Genes with an adjusted $P<0.05$ found by DESeq were identified as differentially expressed.

\section{Gene Validation and Expression Analysis}

Total RNA was isolated from C. kanran buds and flowers using the ZR Plant RNA MiniPrep Kit (Zymo Research, Irvine, CA, USA), and $1 \mu \mathrm{g}$ RNA was used to synthesise oligo(dT)-primed first-strand cDNA by the QuantiTect Reverse Transcription 
Kit (QIAGEN, Hilden, Germany) following the protocol of the manufacturer. The qRT-PCRs were performed in a 7300 Real-Time PCR System (Applied Biosystems, Foster Arthritis 5 City, CA, USA) using an SYBR premix Ex TaqTM Kit (TaKaRa, Kyoto, Japan). The reference gene we chose was actin (c114989.graph_c0). We calculated the relative expression level by the ${ }^{-}{ }^{\Delta} \Delta$ CT method (Livak and Schmittgen, 2001).

\section{Measurement of Flower Flavonoids}

We used the relative quantitative method to analyze flavonoids from white and purple-red C. kanran opened flowers. Approximately $0.1 \mathrm{~g}$ (fresh weight) was extracted in $1 \mathrm{ml}$ $70 \%$ methanol at $4{ }^{\circ} \mathrm{C}$ for 1 day. After centrifugation for $5 \mathrm{~min}$ at $12,000 \mathrm{rpm}$, the supernatant was collected. Before analysis by high-performance liquid chromatography (HPLC), the extract was filtered to remove impurities. Samples were analysed using LC-ESI-QTRAP-MS/MS with an Agilent 1200 Series HPLC system (Agilent Technologies, Waldbronn, Germany) coupled to an Applied Biosystems 4000 QTRAP (Darmstadt, Germany), and quantification of flavonoids was carried out in MRM mode. The analytical conditions were as described previously (Chen et al., 2013). Data acquisition, peak integration, and calculations were performed using Analyst 1.5 software ( $A B$ Sciex, MA, USA). We quantified the anthocyanin and flavonol contents of white, purple-red, and intermediate phenotype C. kanran flowers for Pearson's correlation coefficient in a different way. Approximately, $0.2 \mathrm{~g}$ (fresh weight) was extracted in $1 \mathrm{ml}$ methanol: acetic acid: water (80:2:18, by vol.) at $4^{\circ} \mathrm{C}$ for 1 day. After centrifugation for $5 \mathrm{~min}$ at $12,000 \mathrm{rpm}$, the supernatant was collected, and the pellet was re-extracted for the second time. The combined supernatant was hydrolyzed in boiling water for $1 \mathrm{~h}$ and then quantitated to $2 \mathrm{ml}$. Before analysis by HPLC, the extract was filtered to remove impurities. HPLC was performed as previously described (Wang et al., 2014). Myricetin, kaempferol, quercetin, peonidin, malvidin, delphinidin, pelargonidin, and cyanidin (Sigma-Aldrich, China) were used for qualitative and quantitative analysis. Mean values and SDs were obtained from three replicates.

\section{Construction for Transient Expression by Particle Bombardment}

According to the gene transcript level and flavonoid content, CkCHS-1, CkDFR, and CkANS were screened as key genes encoding enzymes in the flavonoid metabolic pathway of $C$. kanran. Therefore, the three genes were divided into three groups, single genes, double genes, and triple genes, to be validated for transient expression by particle bombardment. The genes driven individually by the $35 \mathrm{~S}$ promoter were cloned into the pCAMBIA1305.1 vector at the NcoI restriction site. The recombinant plasmid DNA was extracted and purified using an EndoFree Plasmid Giga Kit (QIAGEN, Hilden, Germany). Before bombardment, healthy white-type C. kanran flowers were collected, and the following procedure was conducted as described in the study by Ma et al. (2008).
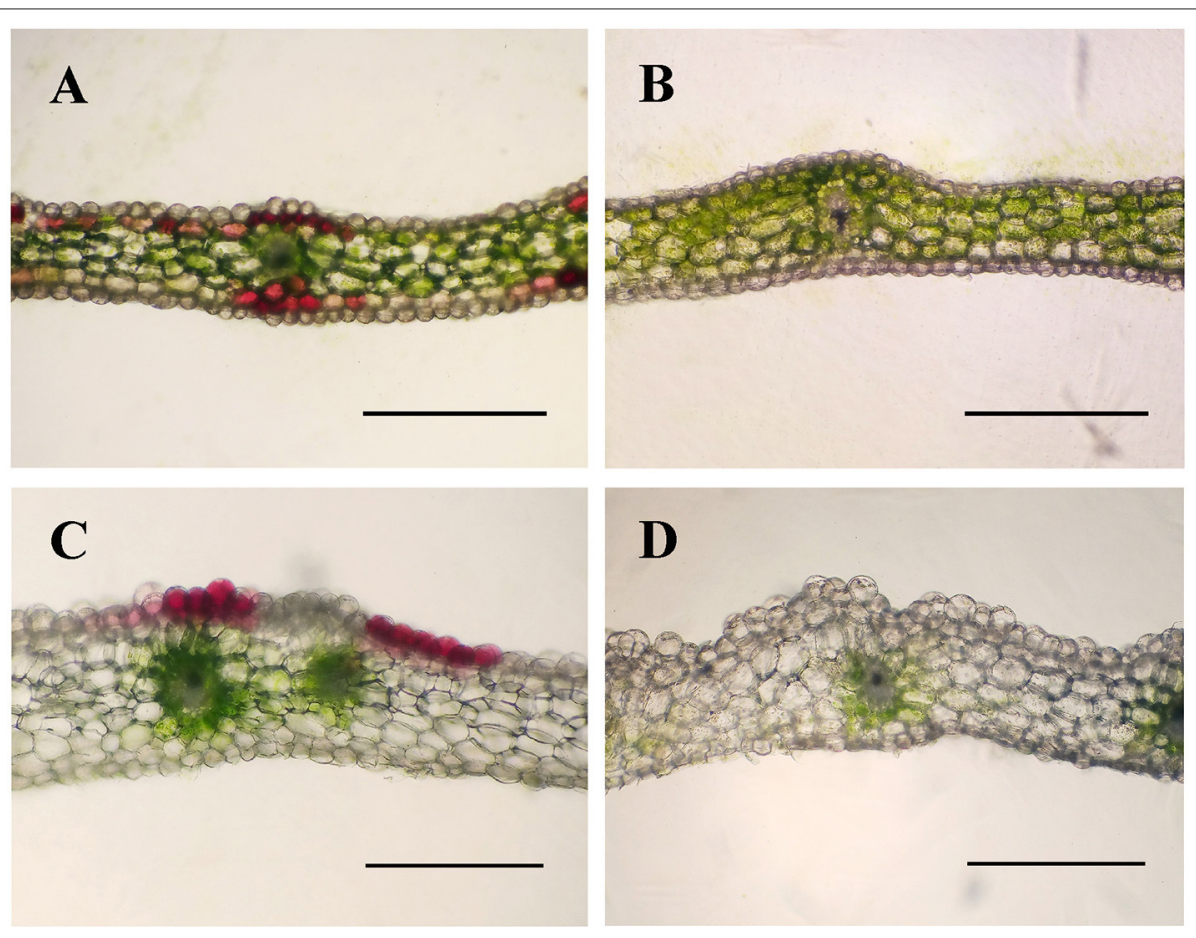

FIGURE 2 | Cross-sections of purple-red and white C. kanran showing the different colour patterns in petals and lips. Anatomic structure of purple-red C. kanran petals (A) and lips (C), white C. kanran petals (B), and lips (D). Scale bar $=500 \mu \mathrm{m}$. 


\section{RESULTS}

\section{Phenotypic Characterisation}

In the process of domestication and cultivation of C. kanran, it is important to note the colour variety of the flowers. The most well-known cultivar, white-type C. kanran, has greenishwhite petals and a white lip, both with greenish stripes (Figures 1A,C-G). Compared with white-type flowers, purplered flowers exhibited purple-red petals with stripes of the same colour and a white lip with red spots and greenish stripes (Figures 1B,H-L). Because of the striking contrast in flower colour, white and purple-red C. kanran are the optimal candidates to reveal the flower coloration mechanism. To study the distribution of anthocyanin production in perianth cells, we analysed petal and lip cross-sections from white and purple-red C. kanran (Figure 2). In the petals, anthocyanins accumulated in the region from subepidermal cells to the xylem (Figure 2A), while in the lip, anthocyanins accumulated only in the epidermal cells of the adaxial surface (Figure 2C). However, white flowers showed no purple-red pigments (anthocyanins) in cells except chlorophyll (Figures 2B,D).

\section{Flavonoid Content}

Anthocyanins, as the largest group of water-soluble pigments, are responsible for most of the purple, blue, and red colours in the plants. In this study, we quantified the flavonoids of purple-red and white C. kanran flowers by a relative quantitative method. In purple-red flowers, only cyanidin, peonidin, and malvidin were present, and the cyanidin content was far greater than that of peonidin and malvidin. Delphinidin and pelargonidintype anthocyanins were not detected, which probably was due to their extremely low content. Interestingly, the contents of myricetin (MY), kaempferol (KA), and quercetin (QU) in the white petals were lower than those in the purple-red C. kanran petals. However, it is worth noting that QU was detected in the white lip at a concentration two times higher than that in the purple-red lip, while the concentrations of MY and KA were similar in both study objects (Figure 3).

\section{RNA-seq, Assembly, and Differential Expression Analysis}

To illustrate the mechanism underlying the variation in flower coloration and to identify potential genes involved in this process, the petals and lips of purple-red and white C. kanran were used to generate four libraries for high-throughput sequencing. After quality cheques, $61.90 \mathrm{~Gb}$ clean data were obtained, and the Q30 percentages (percentage of sequences with sequencing error rates $<0.1 \%$ ) of every sample were higher than $87.72 \%$. These data showed that the sequencing quality and throughput were reliable enough to carry out further research. Short reads were then assembled into 181,335 transcripts and 74,713 unigenes by Trinity with mean lengths of $1,230 \mathrm{bp}$ and $887 \mathrm{bp}$, respectively. After searching the reference sequences using BLASTX against KEGG, COG, KOG, GO, nr, and Swiss-Prot, we obtained a total of 26,930 unigenes providing a significant BLAST result.

Differentially expressed unigenes were grouped into 50 functional categories following KEGG annotation (Figure 4).
A

Flavonoid content in petals ( $\mu \mathrm{g} / \mathrm{g}$ fresh weight)

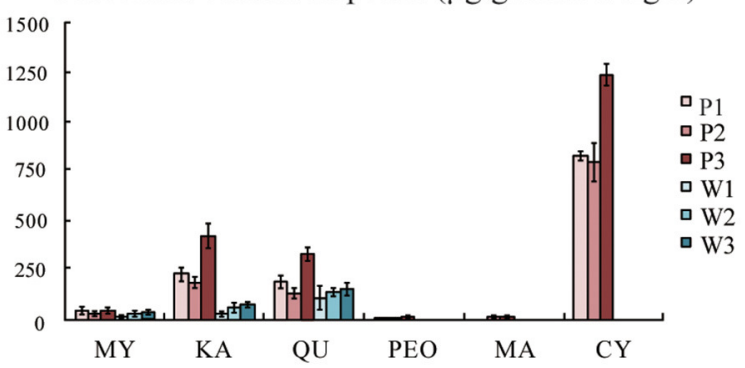

B

Flavonoid content in lips ( $\mu \mathrm{g} / \mathrm{g}$ fresh weight)

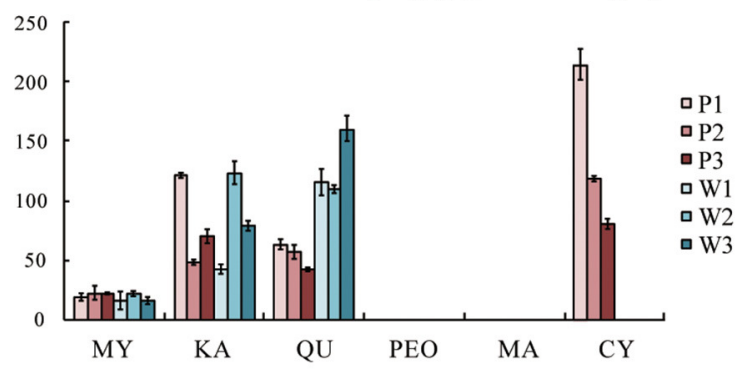

FIGURE 3 | Flavonoid composition analysis by HPLC from flowers of purple-red and white C. kanran. (A) Flavonoid content in petals. (B) Flavonoid content in lips. MY, myricetin; KA, kaempferol; QU, quercetin; PEO, peonidin; MA, malvidin; CY, cyanidin; P1, purple-red C. kanran 1; W1, white C. kanran 1.

The most prevalent functional category was photosynthesis, followed by phenylpropanoid metabolism, which was located upstream of flavonoid metabolism. Members involved in phenylalanine and flavonoid metabolism were also major parts of differentially expressed unigenes. These results indicated that the alternation of flavonoid metabolism was the underlying factor leading to a striking contrast in flower colour between purplered and white C. kanran. Furthermore, the crucial genes taking part in three secondary metabolic pathways (phenylpropanoid, flavonoid, and anthocyanin metabolism referring to KEGG pathways) are listed in Table 1, consisting of 102 unigenes.

To compare levels of pigment biosynthesis-related gene expression, relative expression levels of "petals vs. lips of purplered C. kanran," "petals vs. lips of white C. kanran," "petals of white C. kanran vs. petals of purple-red C. kanran," and "lips of white C. kanran vs. lips of purple-red C. kanran" were conducted. Among the differences in the gene expression profile of white C. kanran vs. purple-red C. kanran, each one of cinnamate 4-hydroxylase $(\mathrm{C} 4 \mathrm{H}), \mathrm{CHS}, \mathrm{DFR}$, and ANS were downregulated, whereas each one of F 3'H, flavonol synthase (FLS), and CCoAOMT were upregulated. In the same flower, two $\mathrm{C} 4 \mathrm{H}$ and each one of CHS, DFR, and ANS were upregulated but only one CHS was downregulated (petals vs. lips of purple-red $C$. kanran), however, there was only one CHS downregulated (petals vs. lips of white C. kanran) (Table 2).

\section{Validating RNA-seq Data by qRT-PCR}

To validate the RNA-seq expression profiles, seven differentially expressed unigenes encoding $\mathrm{C} 4 \mathrm{H}, \mathrm{CHS}, \mathrm{DFR}, \mathrm{ANS}$, and FLS 


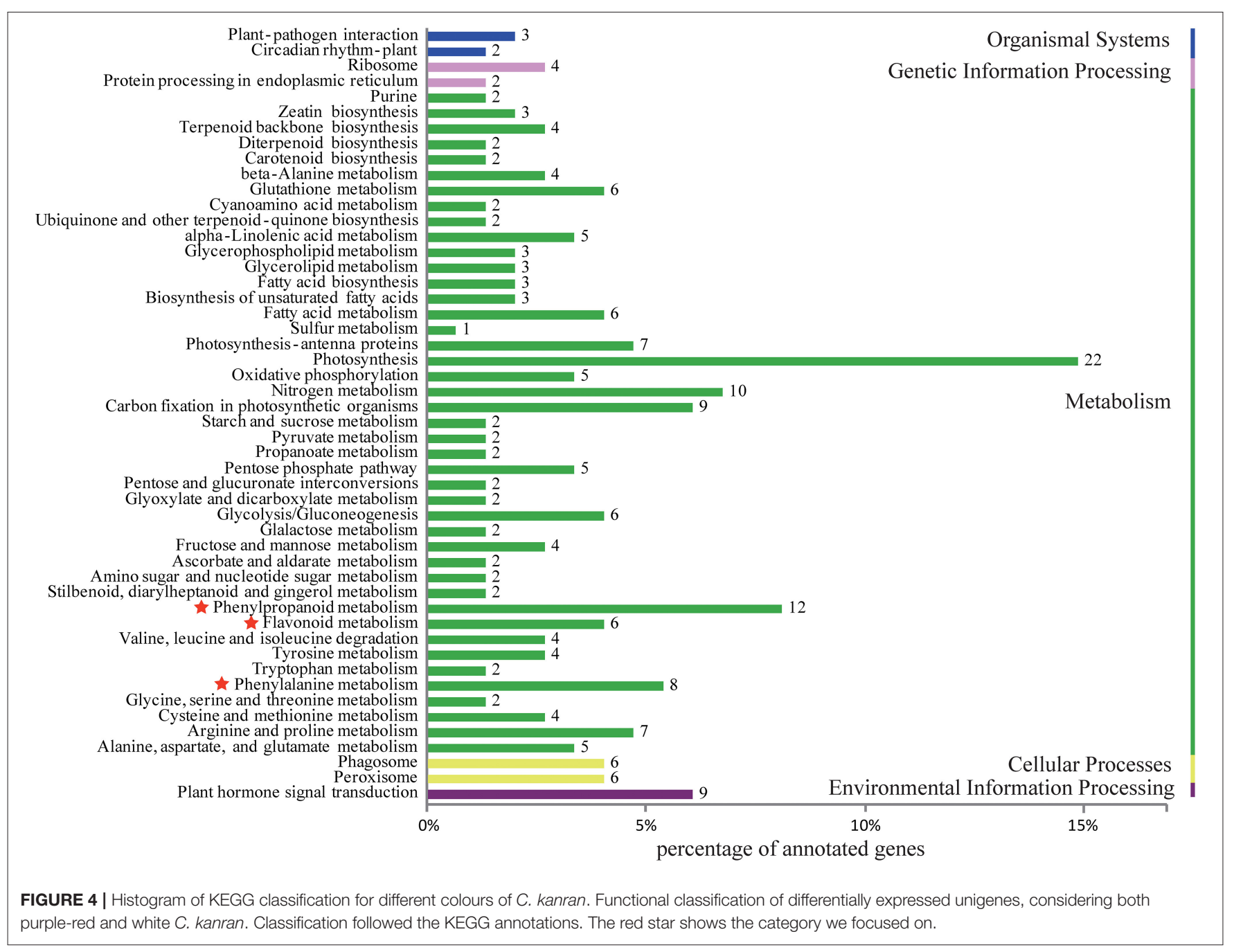

TABLE 1 | Candidate genes related to flower pigmentation of Cymbidium kanran.

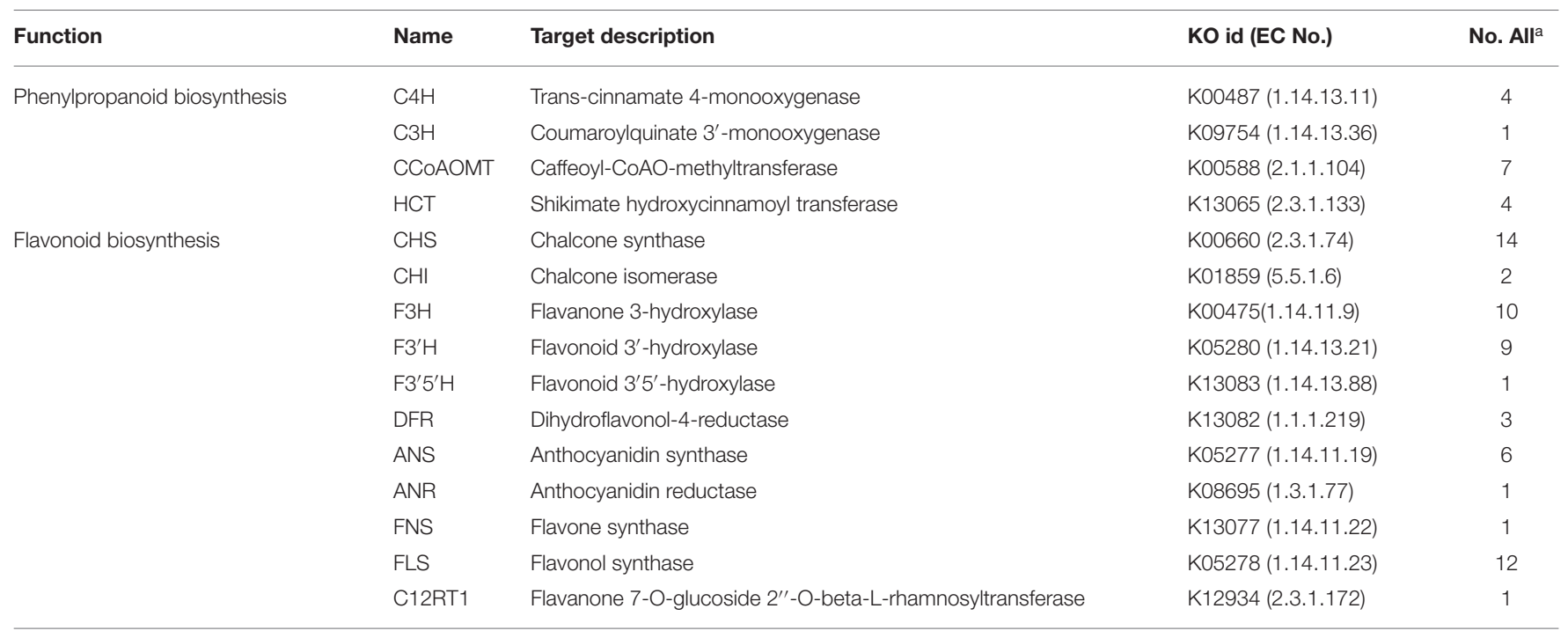


TABLE 1 | Continued

\begin{tabular}{|c|c|c|c|c|}
\hline Function & Name & Target description & KO id (EC No.) & No. All ${ }^{a}$ \\
\hline & FOMT & Flavonol 3-O-methyltransferase & K05279 (2.1.1.76) & 9 \\
\hline \multirow[t]{4}{*}{ Anthocyanin modification } & GT1 & Anthocyanidin 5',3'-O-glucosyltransferase & K12938 (2.4.1.-) & 7 \\
\hline & 3'GT & Anthocyanin 3'-O-beta-glucosyltransferase & K12939 (2.4.1.238) & 1 \\
\hline & $5 \mathrm{MaT1}$ & Anthocyanin 5-O-glucoside-6"' -O-malonyltransferase & K12934 (2.3.1.172) & 1 \\
\hline & UFGT & Anthocyanidin 3-O-glucosyltransferase & K12930 (2.4.1.115) & 5 \\
\hline
\end{tabular}

${ }^{a}$ No. All, the total number of unigenes investigated.

dOmission of numbers for the KO id.

TABLE 2 | Shifts in the expression levels of candidate genes related to flower pigmentation.

\begin{tabular}{|c|c|c|c|c|c|}
\hline Name & Target description & KO id (EC No.) & No. Alla & No. Up ${ }^{b}$ & No. Down ${ }^{c}$ \\
\hline \multicolumn{6}{|c|}{ Petals vs. lips of purple C. kanran } \\
\hline $\mathrm{C} 4 \mathrm{H}$ & Trans-cinnamate 4-monooxygenase & K00487 (1.14.13.11) & 2 & 2 & 0 \\
\hline DFR & Dihydroflavonol 4-reductase & K13082 (1.1.1.219) & 1 & 1 & 0 \\
\hline ANS & Anthocyanidin synthase & K05277 (1.14.11.19) & 1 & 1 & 0 \\
\hline $\mathrm{CHS}$ & Chalcone synthase & K00660 (2.3.1.74) & 1 & 0 & 1 \\
\hline DFR & Dihydroflavonol 4-reductase & K13082 (1.1.1.219) & 1 & 0 & 1 \\
\hline FLS & Flavonol synthase & K05278 (1.14.11.23) & 12 & 1 & 0 \\
\hline ANS & Anthocyanidin synthase & K05277 (1.14.11.19) & 1 & 0 & 1 \\
\hline CCOAOMT & Caffeoyl-CoAO-methyltransferase & K00588 (2.1.1.104) & 1 & 1 & 0 \\
\hline FLS & Flavonol synthase & K05278 (1.14.11.23) & 12 & 1 & 0 \\
\hline
\end{tabular}

${ }^{a}$ No. All, the total number of unigenes investigated.

${ }^{b} \mathrm{No}$. Up, the number of unigenes with higher expression level in the former than the latter.

${ }^{c}$ No. Down, the number of unigenes with lower expression level in the former than the latter.

were quantified by qRT-PCR (Figures 5A-U). The general expression profile obtained by qRT-PCR was perfectly consistent with that obtained by RNA-seq (Figure 5V), supporting the accuracy of the RNA-seq data.

CkC4H-3, CkCHS-1, CkDFR, and CkANS were highly expressed in purple-red floral tissues and expressed at low levels in white floral tissues (Figures 5A-I, M-R). Conversely, CkFLS was expressed at a higher level both in petals and the lip of white C. kanran than purple-red C. kanran (Figures 5S,T). Most of the anthocyanin-related genes exhibited higher expression levels in petals than in the lip, but the opposite results appeared in the CkCHS-2 expression pattern (Figures 5J-L). More interestingly, CkCHS-2 was abundantly expressed in white flowers but expressed at low levels in purplered flowers (Figures 5J,K). Conversely, CkCHS-1 was highly expressed in purple-red flowers but had low expression levels in white flowers (Figures $\mathbf{5 G}, \mathbf{H}$ ), suggesting that white and purple-red flowers may have different CHSs to participate in the flavonoid/anthocyanin biosynthesis pathway. Additionally, CkDFR expression in three shades of purple-red flowers displayed a reverse trend to CkFLS (Figures $5 \mathbf{W}, \mathbf{X}$ ), which is in agreement with the competition to the same substrate between DFR and FLS.

\section{Pearson's Correlation Coefficient Between Gene Transcript Level and Flavonoid Content}

To uncover the relationship between gene expression and flavonoid content, the transcript abundance of flavonoid pathway genes and the accumulation of related products were detected in three shades of flowers (white, purple-red, and intermediate phenotype C. kanran). As presented in Figure 6, 
A

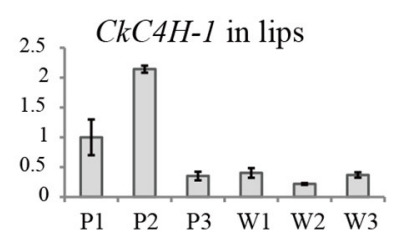

D

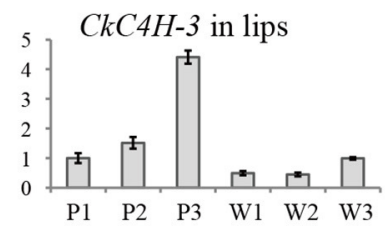

G

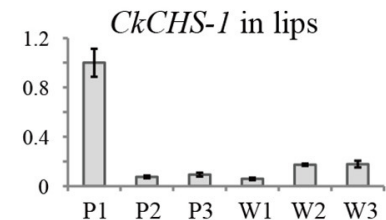

J

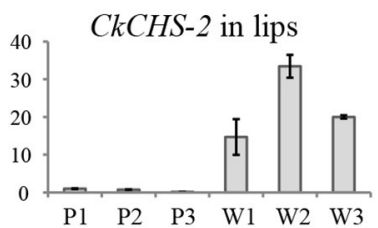

M

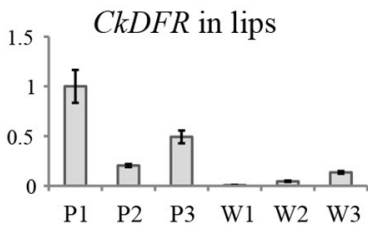

P

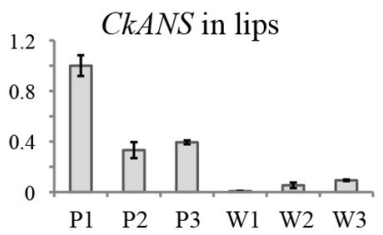

S

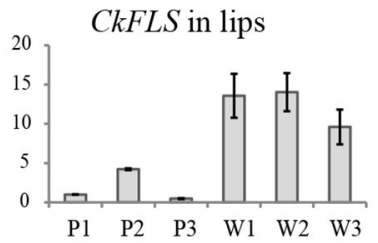

V

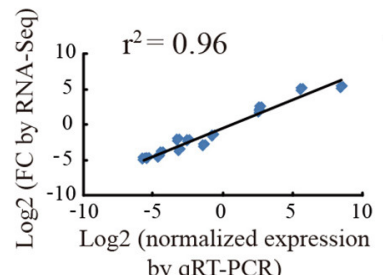

B

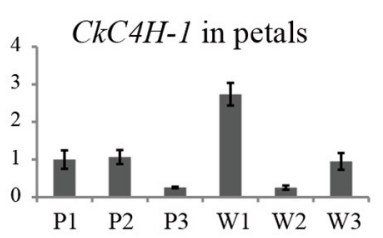

E

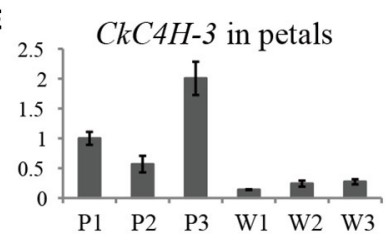

H

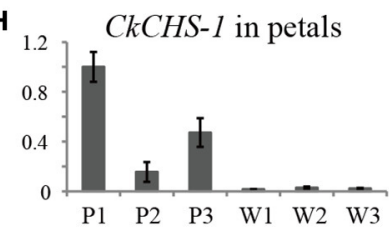

K

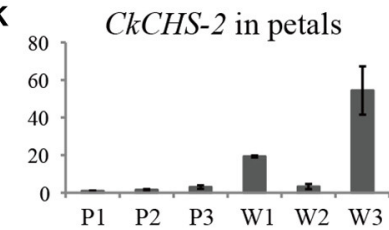

N
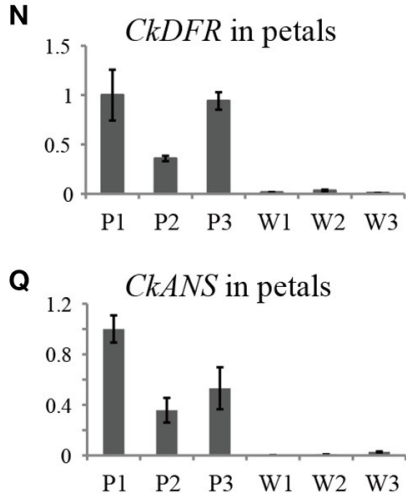

T

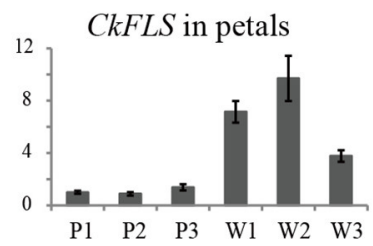

W

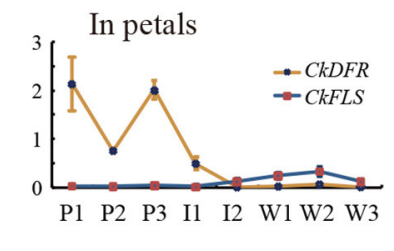

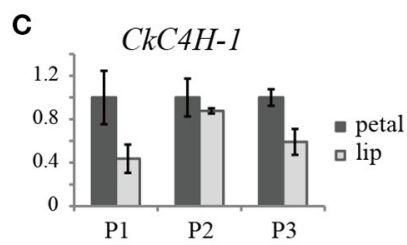

F
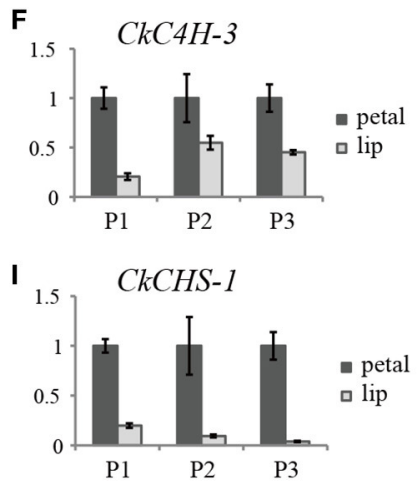

$\mathbf{L}$

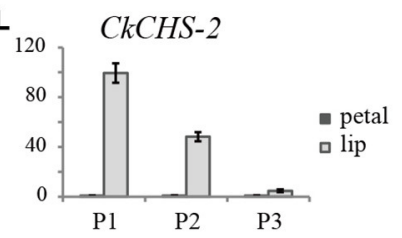

0
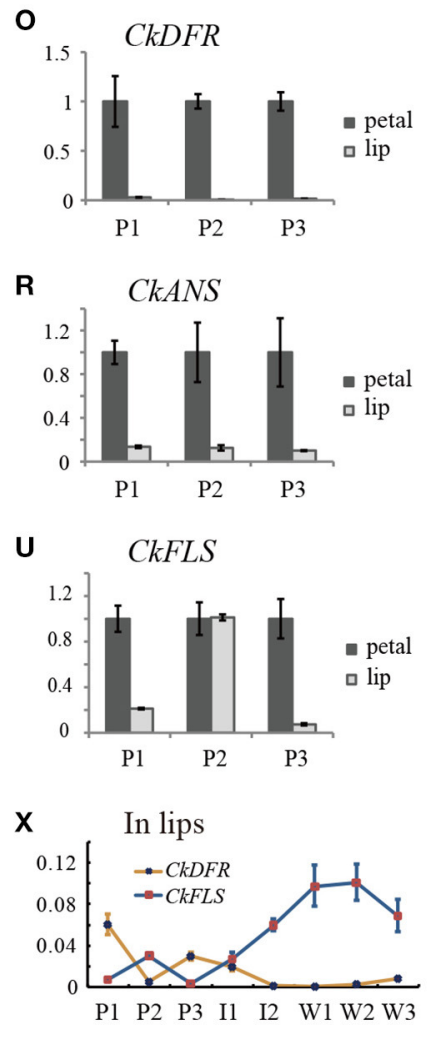

FIGURE 5 | Transcript accumulation measurements of colour-related genes involved in the anthocyanidin metabolic process. (A-U) The qRT-PCR analysis of differentially expressed genes from RNA-Seq analysis. (V) Correlation of gene expression results obtained by qRT-PCR analysis and RNA-Seq for colour-related genes of the purple-red and white flower buds (length of approximately $3.0 \mathrm{~cm}$ ). (W,X) line charts of the relative expression levels of DFR and FLS in petals and lips, respectively. The Y-axis of all charts except V represents the relative expression level. P1, purple-red C. kanran 1; W1, white $C$. kanran 1; I1, intermediate phenotype $C$. kanran 1. 


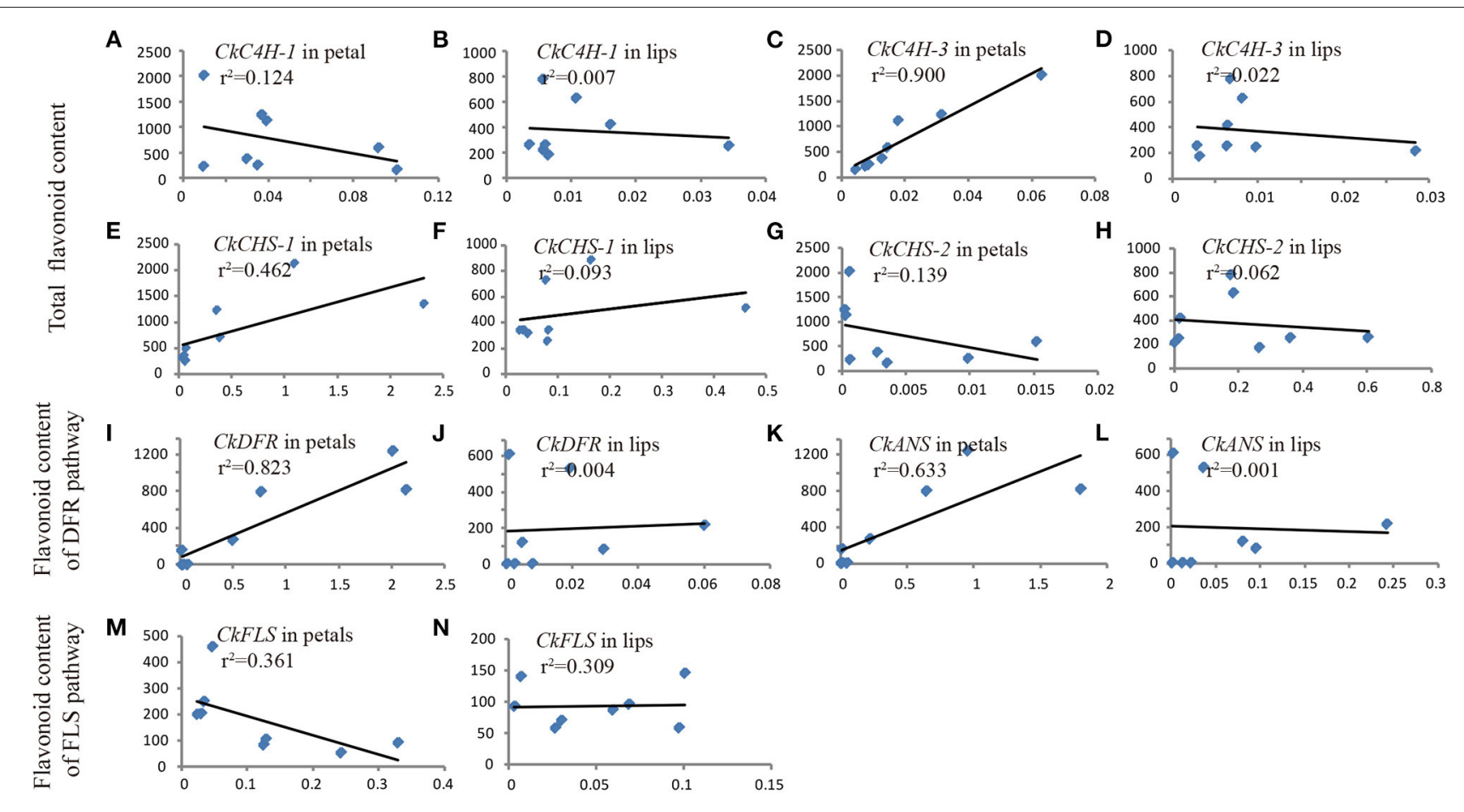

FIGURE 6 | Pearson's correlation coefficient between gene transcript level (X-axis) and flavonoid content (Y-axis) in petals (A,C,E,G,I,K,M) and the lip (B,D,F,H,J,L,N) of C. kanran. Total flavonoids included MY, KA, QU, PEO, MA, and CY. The anthocyanidins of the DFR pathway included PEO, MA, and CY. The flavonol of the FLS pathway included MY, KA, and QU.
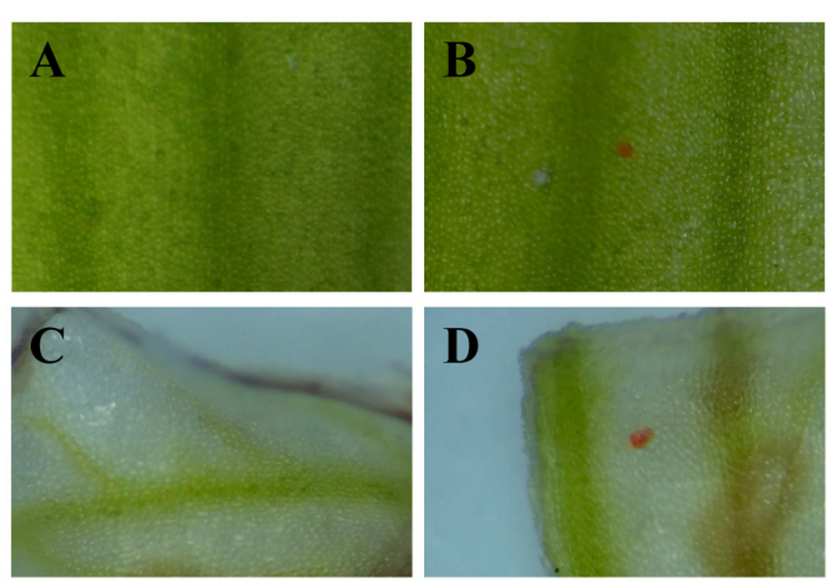

FIGURE 7 | Development of red spots in white petals and lips due to transient expression of CKCHS-1, CKDFR, and CKANS under the control of the 35S promoter. (A) A petal conducted by particle bombardment with empty vector plasmid. (B) A petal conducted by particle bombardment with the mixture of overexpression vector plasmids of the three genes. (C) A lip conducted by particle bombardment with empty vector plasmid. (D) A lip conducted by particle bombardment with the mixture of overexpression vector plasmids of the three genes.

the concentration of flavonoids varied with the expression level of different genes. Normally, two variables show a strong correlation if the correlation coefficient $(r)$ exceeds 0.6 in the Pearson correlation analysis. The contents of total flavonoids were significantly positively correlated with the expression levels of $C k C 4 H-3$ and $C k C H S-1$ in petals $\left(r^{2}=0.9055, r^{2}=0.4642\right)$ (Figures 6C,E). However, there was poor or no correlation between the total flavonoid content and $\mathrm{CkC} 4 \mathrm{H}-1$ and $\mathrm{CkCHS}$ 2 transcripts (Figures 6AB, G,H), which agreed with the qRTPCR results showing ruleless expression levels in purple-red and white C. kanran (Figures 6A-C,J-L). CkDFR and CkANS were involved in the biosynthesis of CY, MA, and PEO and were positively correlated with the anthocyanidin content of the DFR pathway in petals (Figures 6I,L).

\section{Production of Purple-Red Pigmentation in White C. kanran by Transient Expression of CHS-1, DFR, and ANS Genes}

According to Pearson's correlation analysis in Figure 6, we conjectured that the recovery of the CkCHS-1,CkDFR, and CkANS genes in the white type could complete the anthocyanin pathway to display a red pigment similar to that of purple-red C. kanran. Hence, these three genes were divided into three groups, which are single genes, double genes, and triple genes, to be validated for transient expression by particle bombardment. Then, the three groups of genes were bombarded into white petals and lips. After incubation on $1 / 2 \mathrm{MS}$ medium for 5 days, only the group of triple genes accumulated red spots in both petal and lip tissue (Figure 7). This result supported that anthocyanin absence in white-type $C$. kanran probably resulted from the expression deficiency of CkCHS-1, CkDFR, and CkANS. We further evaluated the temporal transcription of these three 

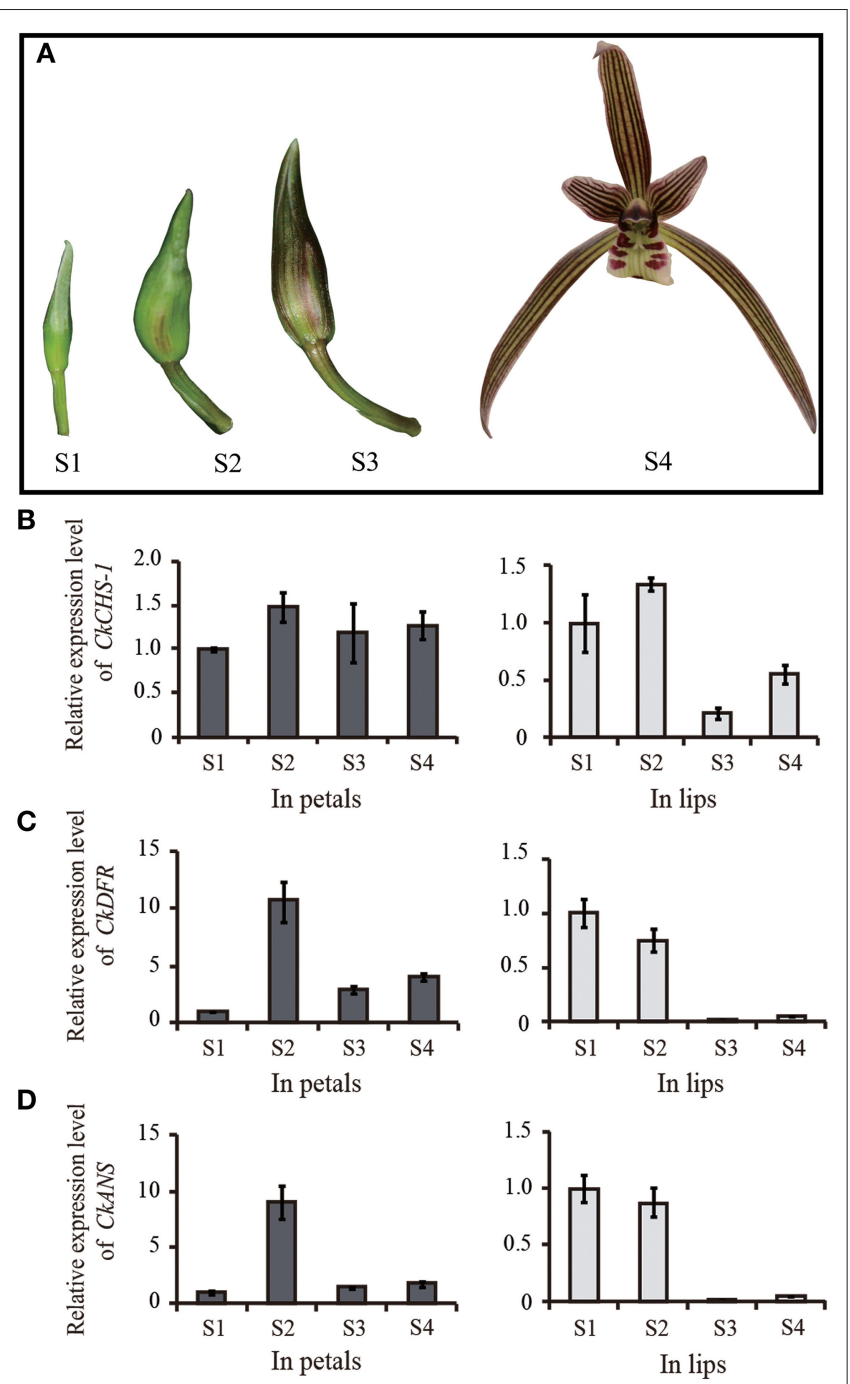

FIGURE 8 | CKCHS-1, CKDFR, and CKANS expression levels were measured by qRT-PCR in four stages. (A) Four development stages of purple-red flower. (B-D) The relative expression level of $C k C H S-1, C k D F R$, and $C K A N S$ in four development stages of petals and lips of purple-red flower, respectively. Four developmental stages: stage 1 ( $\mathrm{S} 1$, bud length of approximately $2 \mathrm{~cm}$ ), stage 2 (S2, bud length of approximately $3 \mathrm{~cm}$ ), stage 3 (S3, 1-2 days before anthesis), and stage 4 (S4, the flower opened).

genes in four developmental stages: stage 1 ( $S 1$, bud length of approximately $2 \mathrm{~cm}$ ), stage 2 (S2, bud length of approximately $3 \mathrm{~cm}$ ), stage 3 (S3, 1-2 days before anthesis), and stage 4 (S4, the flower opened) (Figure 8). In petals, the transcriptional profile showed that CkCHS-1,CkDFR, and CkANS were all actively expressed gradually from $S 1$ to $S 4$ and reached maximal expression in S2. Both CkDFR and CkANS presented a sharp peak at S2, but CkCHS-1 maintained similar transcriptional activity during all stages in petals. These three gene expression patterns in the lip displayed a similar trend of higher expression in early stages $\mathrm{S} 1$ and $\mathrm{S} 2$, while CkDFR and CkANS showed the same trend in both organs.

\section{DISCUSSION}

The main pigments targeted for orchid flowers are anthocyanins that contribute to a variety of colours, such as red, purple, pink, and blue, while studies have shown that the main types of anthocyanidin aglycones in orchids are cyanidin, peonidin, and delphinidin (Liu et al., 2012; Vignolini et al., 2012; Wang et al., 2014; Liang et al., 2020; Zhang et al., 2020). In Oncidium Gower Ramsey, the red portion of lips contained a mixture of cyanidin-3-O-glucoside, delphinidin-3O-glucoside, and peonidin-3-O-glucoside compounds (Liu et al., 2012). Flowers of Phalaenopsis with red-purple, purple, purpleviolet, and violet to violet-blue colour were due to highly accumulated cyanidin-based anthocyanins, but no true-blue colour and no delphinidin was detected (Liang et al., 2020). In some cultivars of Cymbidium hybrids, only cyanidin-, malvidin, and peonidin-type anthocyanins were identified (Woltering and Somhorst, 1990; Johnson et al., 1999). In C. kanran, the flavonoid composition exhibited a sharp shift between purplered and white flowers. Three types (cyanidin, peonidin, and malvidin) of anthocyanidins were also detected in purple-red flowers but were absent in white flowers. While the transcript level of the colour-related gene showed that $\mathrm{CkC} 4 \mathrm{H}-3$, CkCHS1, CkDFR, and CkANS were highly expressed corresponding to purple-red floral tissues compared with white type floral tissues, but CkFLS had a higher level of expression in the white one, and a similar result was also achieved in Pleione limprichtii (Zhang et al., 2020). Dihydroflavonol reductase is a key enzyme in the biosynthesis of anthocyanins and can catalyze the reduction of three colourless dihydroflavonols, DHQ, DHK, and DHM, to leucoanthocyanidins. Because of the similar structure of the three substrates, it is easy to understand that DFRs from many species can utilise all three substrates (Heller et al., 1985; Meyer et al., 1987; Stich et al., 1992; Helariutta et al., 1993; Tanaka et al., 1995; Livak and Schmittgen, 2001; Ma et al., 2008; Chen et al., 2013). dihydroflavonol reductases from some species specifically convert one or two types of dihydroflavonols. In Petunia and Cymbidium, pelargonidin-based brick red/orange flowers do not exist in nature because DFRs cloned from these two species cannot reduce DHK efficiently (Gerats et al., 1982; Forkmann and Ruhnau, 1987; Johnson et al., 1999), suggesting that DFRs have a striking substrate specificity (Springob et al., 2003). In Fragaria species, two DFRs were isolated, and one enzyme variant strongly preferred DHK as a substrate, whereas the other had a higher specificity for DHQ than for DHM (Miosic et al., 2014). Similar results were also reported in Ginkgo biloba and Freesia hybrida (Cheng et al., 2013; Li et al., 2017). In C. kanran, relative quantification showed that cyanidin, peonidin, and malvidin were detected, and the cyanidin content was far greater than that of peonidin and malvidin, indicating that the DFR isolated from C. kanran could catalyze DHQ conversion to leucocyanidin. However, more in vitro enzyme assays are needed to verify the substrate specificity of DFR with DHM and DHK.

Orchid flowers are striking for their specific patterns of colours in the perianths, and the regulation and formation of pigmentation is determined by specific cells of the different floral organs (Yu and Goh, 2001). In the case of C. kanran, the 
distributions of anthocyanin production depended on the tissue specificity. The pigmentation in the petals showed anthocyanins concentrated in the outer layer of subepidermal cells to the xylem (Figure 2A), but in the lip, the red anthocyanins only accumulated in epidermal cells (Figure 2C). Orchid flowers have three petals, and one of the petals is morphologically different in structure and is known as the lip or labellum. However, the differences between the petals and lip were represented not only in shape but also in tissue and genes. The epidermal cells in the lip displayed different forms, in which the adaxial side cells were large and bubble-like, but the abaxial side cells were similar to the petal epidermal cells, which were small and flat. However, anthocyanins accumulated in the region from subepidermal cells to the xylem in the petal (Figure 2A), while in the lip, anthocyanins accumulated only in the epidermal cells of the adaxial surface (Figure 2C). Accordingly, pigments in the adaxial side of lips displayed bright-coloured pigmentation compared with those in petals (Figures 1J,K, 2A,C). Similar results were also reported in the leaves of Tipularia discolour orchid, the same anthocyanins in different anatomical locations led to a broad range of colours (Hughes et al., 2020). In addition, differentially expressed genes in transcriptome analysis of C. kanran supported that the molecular changes also existed between the lip and petals. In petals, CkCHS-1 was highly expressed, but CkCHS-2 was inversely expressed in the lip. In the Petunia hybrid, 12 genes encode CHS from a gene family, which were detected specifically in the margin of the corolla throughout all stages of flower development. Among them, $C H S-A$ and $C H S-J$ were expressed predominantly. These results indicated that $C H S$ expression is regulated by different mechanisms in marginal picotee petals (Saito et al., 2006). According to a previous study, MADSbox genes might collectively regulate the genes involved in colour differentiation in petals and sepals during Cattleya orchid flower development, which may result in the spatiotemporal expression of anthocyanin biosynthesis pathway structural genes to determine colour differentiation (Li et al., 2020).

Knowledge of the molecular genetic basis of the flavonoid biosynthetic pathway allows us to improve the colour quality of plants. The simplest strategy to modify flower colour is to change the amounts of endogenous anthocyanins by regulating the essential enzymes required for flavonoid biosynthesis. For example, transgenic gentian plants with a suppressed ANS showed pale-blue-coloured flowers, while those with a suppressed CHS exhibited a more serious phenotype, i.e., pale-blue to pure white flowers (Nakatsuka et al., 2008). The manipulation of DFR and FLS, which are competing steps for flux towards anthocyanins and flavonols, led to an increase or decrease in anthocyanin content in the flower (Davies and Schwinn, 2010). In addition, the approach of regulating endogenous modification genes has also been utilised

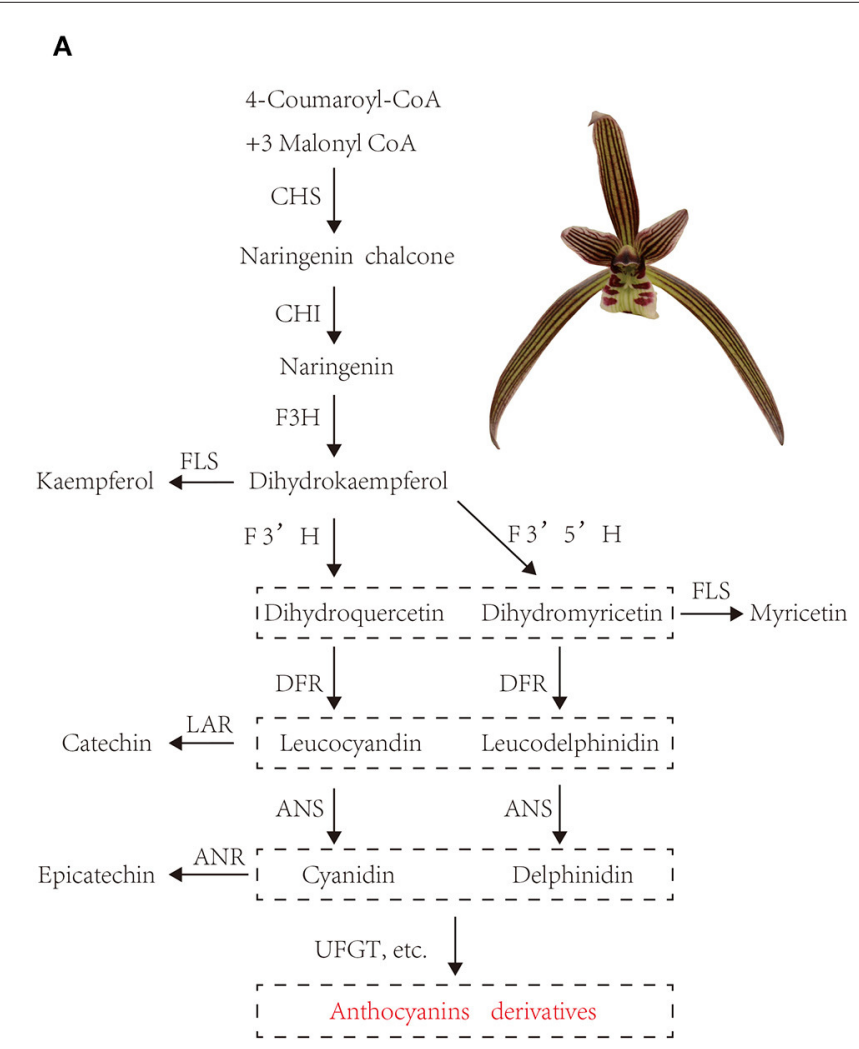

B

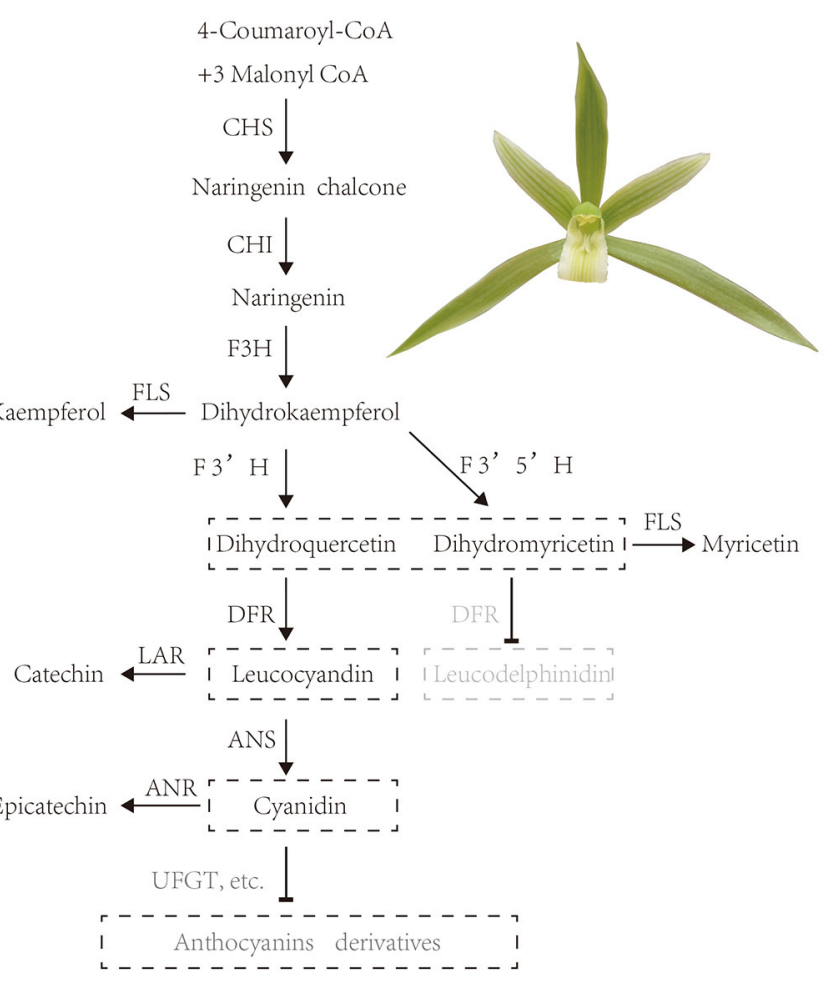

FIGURE 9 | Tentative pathways for (A) purple-red and white (B) C. kanran. CHS, chalcone synthase; $\mathrm{CHI}$, chalcone isomerase; F3H, flavanone 3-hydroxylase; F3'H, flavonoid 3'-hydroxylase; F3'5'H, flavonoid 3'5'-hydroxylase; DFR, dihydroflavonol 4-reductase; ANR, anthocyanidin reductase; ANS, anthocyanidin synthase; FLS, flavonol synthase; LAR, leucoanthocyanidin reductase; UFGT, anthocyanidin 3-O-glucosyltransferase. 
in genetic engineering. For example, F3'5'H suppression by RNAi induced a flower colour change from purple to red in cyclamen (Boase et al., 2010). Similar transformations were also displayed in both F3'5' $\mathrm{H}$ and A5/3'AT (anthocyanin 5,3'-aromaticacyltransferase)-suppressed transgenic gentian plants (Nishihara and Nakatsuka, 2010). In C. kanran, the validation of the transient expression of key genes involved in colour formation showed that only triple genes, CkCHS-1, $C k D F R$, and CkANS could accumulate red spots in both the petal and lip tissue, indicating that the regulatory mechanism of coloration is complicated. To date, studies on some genetically defined genotypes or mutants revealing blocks of anthocyanin pathway gene expression have already been verified in many plants. In Forsythia, it was shown to be blocked in anthers at both the DFR and ANS levels but at the ANS level, only in petals (Rosati et al., 1999). However, the absence of anthocyanins in the skins of white berry varieties is due to a block only of UFGT expression in Vitis (Boss et al., 1996). Additionally, a point mutation of the CHS gene could result in the formation of the white flower of Matthiola incana (Hemleben et al., 2004). Another study in Oncidium also revealed that the failure of anthocyanin accumulation may be due to the inactivation of $\mathrm{OgCHS}$ caused by epigenetic methylation of the 5'-upstream promoter regions (Liu et al., 2012). However, the silencing of three different CHS genes of the purple-colour flower of Dianthus chinensis via VIGS could obtain white or pale purple flower (Liu et al., 2021), suggesting that several CHS genes may be involved in the formation of coloration. In C. kanran, it is noteworthy that $\mathrm{CkCHS}-2$ was abundantly expressed in white flowers but expressed at low levels in purple-red flowers (Figures 5J,K), and CkCHS-1 was highly expressed in purplered flowers but had lower expression levels in white flowers (Figures 5G,H), indicating the complicated regulation of CHSs in C. kanran. Therefore, further studies need to be performed on the regulatory mechanism of anthocyanin pathway-related genes in C. kanran.

Thus, we tentatively speculated about the pathways for purple-red and white C. kanran (Figure 9). In purple-red $C$. kanran, flavonoids are initially derived from phenylalanine and phenylpropanoid metabolism. Subsequently, CkCHS is generated to produce chalcones, and $C k C H I$ catalyzes chalcones into naringenin. Furthermore, naringenin can be converted through $\mathrm{CkF3H}, \mathrm{CkF3}$ ' $\mathrm{H}$, and $\mathrm{CkF3}{ }^{\prime}{ }^{\prime} \mathrm{H}$ to produce dihydroxyflavonols including DHK, DHQ, and DHM. Following the above reaction, $C k D F R$ and $C k A N S$ further catalyze the divergent conversion of dihydroflavonols to produce colourless cyanidin and delphinidin. In the end, CkUFGT and other GTs catalyze the glucosylation of anthocyanidins (peonidin, malvidin, and cyanidin). As for white C. kanran, the main divergence of flavonoid metabolism is anthocyanin metabolism, the GTs cannot be regulated and fulfilled to catalyze the glucosylation of anthocyanidins. Whitetype flowers of Cymbidium are uncommon in nature, and traditional division propagation requires a long period, which leads to a low reproductive rate and high price. In this study, the molecular mechanism of anthocyanidin deficiency in whitetype flowers establishes a foundation for genetic engineering to generate white-type C. kanran. The simplest strategy is to suppress the expression levels of CkCHS-1, CkDFR, and CkANS.
Concurrently, the regulatory mechanism of genes involved in the anthocyanin metabolic pathway has also been carried out in orchids. In Oncidium, OgMYB1 directly activated the expression of $\mathrm{OgCHI}$ and $\mathrm{OgDFR}$ to regulate anthocyanin accumulation in the lip (Chiou and Yeh, 2008). In Phalaenopsis, three MYB transcription factors determine full-red pigmentation, red spots, and venation patterns (Hsu et al., 2015). A recent study in $C$. hybrids also indicated that CyMYB1 regulates temporal- and temperature-dependent anthocyanin accumulation in petals (Nakatsuka et al., 2019). In C. kanran, we also isolated several differentially expressed $M Y B$ genes by Biochemical and Biophysical Research Communications transcriptome analysis and attempted to reveal the molecular mechanism of MYB genes regulating pigmentation patterning, which may provide genetic resources for C. kanran molecular breeding in the future.

\section{CONCLUSIONS}

The white and purple-red phenotype C. kanran flowers had provided a unique experimental system to examine the mechanism for flower anthocyanin pigmentation. Compared with white flowers, purple-red flowers are comprised of three types of anthocyanidin compounds, namely, peonidin, malvidin, and cyanidin, which were shown by HPLC analysis. A total of 102 candidate genes involved in the flower coloration of C. kanran were identified using transcriptome analysis. Gene expression profiles showed that four of these genes were positively correlated with anthocyanidin biosynthesis by qRT-PCR. Transient expression of CkCHS-1,CkDFR, and CkANS by particle bombardment results in the accumulation of anthocyanins in white flowers. These results indicated that CkCHS-1, CkDFR, and CkANS are the key genes involved in floral pigment accumulation in C. kanran. This study also provided a platform for C. kanran functional genomic research.

\section{DATA AVAILABILITY STATEMENT}

The datasets presented in this study can be found in online repositories. The name of the repository and accession number can be found below: National Centre for Biotechnology Information (NCBI) BioProject, https://www.ncbi.nlm.nih.gov/ bioproject/, PRJNA744371.

\section{AUTHOR CONTRIBUTIONS}

ZZ, XW, and ZL designed the experiments. ZZ, XW, YY, and ZY performed all experiments. ZZ, XW, and ZW analysed the data. $\mathrm{ZZ}$ and XW wrote the manuscript. ZL revised the manuscript. All the authors have read and agreed to the published version of the manuscript.

\section{FUNDING}

This research was funded by the Natural Science Foundation of Zhejiang Province (grant nos. 
LY20C160005 and LY19C150003), National Key R \& D Program of China (grant nos. 2018YFD1000401 and 2019YFD1000400), and Wenzhou Agricultural New Variety Breeding Cooperative Group Project (grant no. 2019ZX004-3).

\section{REFERENCES}

Aguiar, J., Giurfa, M., and Sazima, M. (2020). A cognitive analysis of deceptive pollination: associative mechanisms underlying pollinators' choices in non-rewarding colour polymorphic scenarios. Sci. Rep. 10:9476. doi: 10.1038/s41598-020-66356-4

Altschul, S. F., Madden, T. L., Schäffer, A. A., Zhang, J., Zhang, Z., Miller, W., et al. (1997). Gapped BLAST and PSI BLAST: a new generation of protein database search programs, Nucleic. Acids. Res. 25, 3389-3402. doi: $10.1093 /$ nar/25.17.3389

Basist, G., Dyer, A. G., Garcia, J. E., Raleigh, R. E., and Lawrie, A. C. (2021). Why variation in flower color may help reproductive success in the endangered Australian orchid Caladenia fulva. Front. Plant Sci. 12, 874. doi: 10.3389/fpls.2021.599874

Boase, M. R., Lewis, D. H., Davies, K. M., Marshall, G. B., Patel, D., Schwinn, K. E., et al. (2010). Isolation and antisense suppression of flavonoid 3', 5'hydroxylase modifies flower pigments and color in cyclamen. BMC Plant Biol. 10:107. doi: 10.1186/1471-2229-10-107

Boss, P. K., Davies, C. H., and Robinson, S. P. (1996). Expression of anthocyanin biosynthesis pathway genes in red and white grapes. Plant Mol. Biol. 32, 565-569. doi: 10.1007/BF00019111

Chen, S. C., and Tang, T. A. (1982). "A general review of the orchid flora of China," in Orchid Biology: Review and Perspectives, Vol. II, ed J. Arditti (New York, NY: Cornell University Press), 39-87.

Chen, W., Gong, L., Guo, Z., Wang, W., Zhang, H., Liu, X., et al. (2013). A novel integrated method for large-scale detection, identification, and quantification of widely targeted metabolites: application in the study of rice metabolomics. Mol. Plant. 6:1769. doi: 10.1093/mp/sst080

Chen, W. H., Hsu, C. Y., Cheng, H. Y., Chang, H., Chen, H. H., and Ger, M. J. (2011). Downregulation of putative UDP-glucose: flavonoid 3-Oglucosyltransferase gene alters flower coloring in Phalaenopsis. Plant Cell Rep. 30, 1007-1017. doi: 10.1007/s00299-011-1006-1

Cheng, H., Li, L., Cheng, S., Cao, F., Xu, F., Yuan, H., et al. (2013). Molecular cloning and characterization of three genes encoding dihydroflavonol-4reductase from Ginkgo biloba in anthocyanin biosynthetic pathway. PLoS ONE 8:e72017. doi: 10.1371/journal.pone.0072017

Chiou, C. Y., and Yeh, K. W. (2008). Differential expression of MYB gene $(O g M Y B 1)$ determines color patterning in floral tissue of Oncidium Gower Ramsey. Plant Mol. Biol. 66, 379-388. doi: 10.1007/s11103-00 7-9275-3

Davies, K. M., and Schwinn, K. E. (2010). "Molecular biology and biotechnology of flower pigments," in Plant Developmental Biology-Biotechnological Perspectives, ed E. Pua and M. Davey, (Berlin, Heidelberg: Springer), 161-187.

Du Puy, D., Cribb, P., and Tibbs, M. (2007). The genus Cymbidium. Kew: Royal Botanic Gardens Kew.

Forkmann, G., and Ruhnau, B. (1987). Distinct substrate specificity of dihydroflavonol-4-reductase from flowers of Petunia hybrid. Z. Naturforsch. C. 42, 1146-1148. doi: 10.1515/znc-1987-9-1026

Gerats, A. G., Vlaming, P. D., Doodeman, M., Al, B., and Schram, A. W. (1982). Genetic control of the conversion of dihydroflavonols into flavonols and anthocyanins in flowers of Petunia hybrid. Planta 155, 364-368. doi: 10.1007/BF00429466

Gigord, L. D. B., Macnair, M. R., and Smithson, A. (2001). Negative frequencydependent selection maintains a dramatic flower color polymorphism in the rewardless orchid Dactylorhiza sambucina (L.) Soò. Proc. Natl. Acad. Sci. U.S.A. 98, 6253-6255. doi: 10.1073/pnas.111162598

Grabherr, M. G., Haas, B. J., Yassour, M., Levin, J. Z., Thompson, D. A., Amit, I., et al. (2011). Full-length transcriptome assembly from RNA-Seq data without a reference genome. Nat. Biotechnol. 29:644. doi: 10.1038/nbt.1883

\section{ACKNOWLEDGMENTS}

We would like to thank Biogle Gene Tech. Co., Ltd., and Dr. Jinda Wang (Fujian Agriculture and Forestry University) for the help with particle bombardment experimentation.

Grotewold, E. (2006). The genetics and biochemistry of floral pigments. Annu. Rev Plant Biol. 57, 761-780. doi: 10.1146/annurev.arplant.57.032905.105248

Han, Y. Y., Ming, F., Wang, W., Wang, J. W., Ye, M. M., and Shen, D. L. (2006). Molecular evolution and functional specialization of chalcone synthase superfamily from Phalaenopsis orchid. Genetica 128, 429-438. doi: 10.1007/s10709-006-7668-x

Hatier, J. H. B., and Gould, K. S. (2007). Black coloration in leaves of Ophiopogon planiscapus 'Nigrescens'. Leaf optics, chromaticity, and internal light gradients. Funct. Plant Biol. 34, 130-138. doi: 10.1071/FP06220

Helariutta, Y., Elomaa, P., Kotilainen, M., Seppanen, P., and Teeri, T. H. (1993). Cloning of cDNA coding for dihydroflavonol-4-reductase (DFR) and characterization of $d f r$ expression in the corollas of Gerbera hybrida var. regina (Compositae). Plant Mol. Biol. 22, 183-193. doi: 10.1007/BF00014927

Heller, W., Forkmann, G., Britsch, L., and Grisebach, H. (1985). Enzymatic reduction of (+)-dihydroflavonols to flavan-3,4-cis-diols with flower extracts from Matthiola incana and its role in anthocyanin biosynthesis. Planta 165, 284-287. doi: 10.1007/BF00395052

Hemleben, V., Dressel, A., Epping, B., Lukačin, R., Martens, S., and Austin, M. (2004). Characterization and structural features of a chalcone synthase mutation in a white-flowering line of Matthiola incana R. Br. (Brassicaceae). Plant Mol. Biol. 55, 455-465. doi: 10.1007/s11103-004-1125-y

Hieber, A. D., Mudalige-Jayawickrama, R. G., and Kuehnle, A. R. (2006). Color genes in the orchid Oncidium Gower Ramsey: identification, expression, and potential genetic instability in an interspecific cross. Planta 223, 521-531. doi: 10.1007/s00425-005-0113-Z

Hsiao, Y. Y., Pan, Z. J., Hsu, C. C., Yang, Y. P., Hsu, Y. C., Chuang, Y. C., et al. (2011). Research on orchid biology and biotechnology. Plant Cell Physiol. 52, 1467-1486. doi: 10.1093/pcp/pcr100

Hsu, C. C., Chen, Y. Y., Tsai, W. C., Chen, W. H., and Chen, H. H. (2015). Three R2R3-MYB transcription factors regulate distinct floral pigmentation patterning in Phalaenopsis spp. Plant Physiol. 168, 175-191. doi: 10.1104/pp.114.254599

Hughes, N. M., Connors, M. K., Grace, M. H., Lila, M. A., and Wommack, A. J. (2020). The same anthocyanins served four different ways: insights into anthocyanin structure-function relationships from the wintergreen orchid, Tipularia discolor. Plant Sci. 303:110793. doi: 10.1016/j.plantsci.2020.110793

Johnson, E. T., Yi, H., Shin, B., Oh, B. J., Cheong, H., and Choi, A. G. (1999). Cymbidium hybrida dihydroflavonol 4-reductase does not efficiently reduce dihydrokaempferol to produce orange pelargonidin-type anthocyanins. Plant J. 19, 81-85. doi: 10.1046/j.1365-313X.1999.00502.x

Katsumoto, Y., Fukuchi-Mizutani, M., Fukui, Y., Brugliera, F., Holton, T. A., Karan, M., et al. (2007). Engineering of the rose flavonoid biosynthetic pathway successfully generated blue-hued flowers accumulating delphinidin. Plant Cell Physiol. 48, 1589-1600. doi: 10.1093/pcp/pcm 131

Li, B., and Dewey, C. N. (2011). RSEM: accurate transcript quantification from RNA-Seq data with or without a reference genome. BMC Bioinformatics 12:323. doi: 10.1186/1471-2105-12-323

Li, B. J., Zheng, B. Q., Wang, J. Y., Tsai, W. C., Lu, H. C., Zou, L. H., et al. (2020). New insight into the molecular mechanism of colour differentiation among floral segments in orchids. Commun. Biol. 3:89. doi: 10.1038/s42003-020-0821-8

Li, H., Liu, J., Pei, T., Bai, Z., Han, R., and Liang, Z. (2019). Overexpression of SmANS enhances anthocyanin accumulation and alters phenolic acids content in Salvia miltiorrhiza and Salvia miltiorrhiza Bge f. alba plantlets. Int. J. Mol. Sci. 20, 2225. doi: 10.3390/ijms20092225

Li, Q., Wang, J., Sun, H. Y., and Shang, X. (2014). Flower color patterning in pansy (Viola $\times$ wittrockiana Gams.) is caused by the differential expression of three genes from the anthocyanin pathway in acyanic and cyanic flower areas. Plant Physiol. Biochem. 84, 134-141. doi: 10.1016/j.plaphy.2014.09.012 
Li, Y., Liu, X., Cai, X., Shan, X., Gao, R., Yang, S., et al. (2017). Dihydroflavonol 4-reductase genes from Freesia hybrida play important and partially overlapping roles in the biosynthesis of flavonoids. Front. Plant Sci. 8:428. doi: 10.3389/fpls.2017.00428

Liang, C. Y., Rengasamy, K. P., Huang, L. M., Hsu, C. C., Jeng, M. F., Chen, W. H., et al. (2020). Assessment of violet-blue color formation in Phalaenopsis orchids. BMC Plant Biol. 20:212. doi: 10.1186/s12870-020-02402-7

Liu, J., Hao, X. L., and He, X. Q. (2021). Characterization of three chalcone synthase-like genes in Dianthus chinensis. Plant Cell, Tissue Organ Cult. 146, 483-492. doi: 10.1007/s11240-021-02081-8

Liu, X. J., Chuang, Y. N., Chiou, C. Y., Chin, D. C., Shen, F. Q., and Yeh, K. W. (2012). Methylation effect on chalcone synthase gene expression determines anthocyanin pigmentation in floral tissues of two Oncidium orchid cultivars. Planta 236, 401-409. doi: 10.1007/s00425-012-1616-z

Livak, K. J., and Schmittgen, T. D. (2001). Analysis of relative gene expression data using real-time quantitative PCR and the 2 (-Delta Delta C (T)) method. Methods 25, 402-408. doi: 10.1006/meth.2001.1262

Ma, H., Pooler, M., and Griesbach, R. (2008). Ratio of Myc and Myb transcription factors regulates anthocyanin production in orchid flowers. J. Am. Soc. Hortic. Sci. 133, 133-138. doi: 10.21273/JASHS.133.1.133

Meyer, P., Heidmann, I., Forkmann, G., and Saedler, H. (1987). A new petunia flower colour generated by transformation of a mutant with a maize gene. Nature 330, 677-678. doi: 10.1038/330677a0

Miosic, S., Thill, J., Milosevic, M., Gosch, C., Pober, S., Molitor, C., et al. (2014). Dihydroflavonol 4-reductase genes encode enzymes with contrasting substrate specificity and show divergent gene expression profiles in Fragaria species. PLoS ONE 9:e112707. doi: 10.1371/journal.pone.0112707

Moreau, C., Hill, L., Ellis, T. N., and Hofer, J. M. (2012). The $b$ gene of pea encodes a defective flavonoid $35^{\prime}$-hydroxylase, and confers pink flower color. Plant Physiol. 159, 759-768. doi: 10.1104/pp.112.197517

Mudalige-Jayawickrama, R. G., Champagne, M. M., Hieber, A. D., and Kuehnle, A. R. (2005). Cloning and characterization of two anthocyanin biosynthetic genes from Dendrobium orchid. J. Am. Soc. Hortic. Sci. 130, 611-618. doi: 10.21273/JASHS.130.4.611

Nakatsuka, T., Kei-Ichiro, M., Abe, Y., Kubota, A., Kakizaki, Y., Yamamura, S., et al. (2008). Flower color modification of gentian plants by RNAi-mediated gene silencing. Plant Biotechnol. 25, 61-68. doi: 10.5511/plantbiotechnology.25.61

Nakatsuka, T., Suzuki, T., Harada, K., Kobayashi, Y., Dohra, H., and Ohno, H. (2019). Floral organ- and temperature-dependent regulation of anthocyanin biosynthesis in Cymbidium hybrid flowers. Plant Sci. 287, 110173. doi: $10.1016 /$ j.plantsci.2019.110173

Nishihara, M., and Nakatsuka, T. (2010). "Genetic engineering of novel flower colors in floricultural plants: recent advances via transgenic approaches," in Protocols for In Vitro Propagation of Ornamental Plant, ed S. Jain and S. Ochatt (Totowa, NJ: Humana Press), 325-347.

Passeri, V., Koes, R., and Quattrocchio, F. M. (2016). New challenges for the design of high value plant products: stabilization of anthocyanins in plant vacuoles. Front. Plant Sci. 7:153. doi: 10.3389/fpls.2016.00153

Petroni, K., and Tonelli, C. (2011). Recent advances on the regulation of anthocyanin synthesis in reproductive organs. Plant Sci. 181, 219-229. doi: 10.1016/j.plantsci.2011.05.009

Ramazzotti, S., Filippetti, I., and Intrieri, C. (2008). Expression of genes associated with anthocyanin synthesis in red-purplish, pink, pinkish-green and green grape berries from mutated 'Sangiovese' biotypes: a case study. Vitis 47, 147-151. doi: 10.5073/vitis.2008.47.147-151

Rosati, C., Cadic, A., Duron, M., Ingouff, M., and Simoneau, P. (1999). Molecular characterization of the anthocyanidin synthase gene in Forsythia intermedia reveals organ-specific expression during flower development. Plant Sci. 149, 73-79. doi: 10.1016/S0168-9452(99)00146-6

Saito, R., Fukuta, N., Ohmiya, A., Itoh, Y., Ozeki, Y., Kuchitsu, K., et al. (2006). Regulation of anthocyanin biosynthesis involved in the formation of marginal picotee petals in Petunia. Plant Sci. 170, 828-834. doi: $10.1016 /$ j.plantsci.2005.12.003
Singh, D. R., Pamarthi, R. K., Kumar, R., Rai, D., Meitei, A. L., and Kiran Babu, P. (2019). Traditional artifacts from dried leaves of Cymbidium species (Orchidaceae) in Indian state of Sikkim. Indian J. Tradit. Knowl. 18, 390-394. http://nopr.niscair.res.in/handle/123456789/47067

Springob, K., Nakajima, J., Yamazaki, M., and Saito, K. (2003). Recent advances in the biosynthesis and accumulation of anthocyanins. Nat. Prod. Rep. 20, 288-303. doi: 10.1039/b109542k

Stich, K., Eidenberger, T., Wurst, F., and Forkmann, G. (1992). Enzymatic conversion of dihydroflavonols to flavan-3,4-diols using flower extracts of Dianthus caryophyllus L. (carnation). Planta. 187, 103-108. doi: 10.1007/BF00201630

Tanaka, Y., Fukui, Y., Fukuchi-Mizutani, M., Holton, T. A., Higgins, E., and Kusumi, T. (1995). Molecular cloning and characterization of Rosa hybrida dihydroflavonol 4-reductase gene. Plant Cell Physiol. 36, 1023-1031. doi: 10.1093/oxfordjournals.pcp.a078844

Tanaka, Y., Sasaki, N., and Ohmiya, A. (2008). Biosynthesis of plant pigments: anthocyanins, betalains, and carotenoids. Plant J. 54, 733-749. doi: 10.1111/j.1365-313X.2008.03447.x

Vignolini, S., Davey, M. P., Bateman, R. M., Rudall, P. J., Moyroud, E., Tratt, J., et al. (2012). The mirror crack'd: both pigment and structure contribute to the glossy blue appearance of the mirror orchid, Ophrys speculum. New Phytol. 196, 1038-1047. doi: 10.1111/j.1469-8137.2012.0 4356.x

Wang, L., Albert, N. W., Zhang, H., Arathoon, S., Boase, M. R., Ngo, H., et al. (2014). Temporal and spatial regulation of anthocyanin biosynthesis provide diverse flower color intensities and patterning in Cymbidium orchid. Planta 240, 983-1002. doi: 10.1007/s00425-014-2152-9

Woltering, E. J., and Somhorst, D. (1990). Regulation of anthocyanin synthesis in Cymbidium flowers: effects of emasculation and ethylene. J. Plant Physiol. 136, 295-299. doi: 10.1016/S0176-1617(11)80052-0

Yu, H., and Goh, C. J. (2001). Molecular genetics of reproductive biology in orchids. Plant Physiol. 127, 1390-1393. doi: 10.1104/pp.127.4.1390

Yu, Z., Liao, Y., Teixeira da Silva, J., Yang, Z., and Duan, J. (2018). Differential accumulation of anthocyanins in Dendrobium officinale stems with red and green peels. Int. J. Mol. Sci. 19:2857. doi: 10.3390/ijms19 102857

Zhang, Y. Y., Zhou, T. H., Dai, Z. W., Dai, X. Y., Li, W., Cao, M. X., et al. (2020). Comparative transcriptomics provides insight into floral color polymorphism in a Pleione limprichtii orchid population. Int. J. Mol. Sci. 21:247. doi: 10.3390/ijms21010247

Zhao, Z. C., Hu, G. B., Hu, F. C., Wang, H. C., Yang, Z. Y., and Lai, B. (2012). The UDP glucose: flavonoid-3-O-glucosyltransferase (UFGT) gene regulates anthocyanin biosynthesis in litchi (Litchi chinesis Sonn.) during fruit coloration. Mol. Biol. Rep. 39, 6409-6415. doi: 10.1007/s11033-011-1303

Conflict of Interest: The authors declare that the research was conducted in the absence of any commercial or financial relationships that could be construed as a potential conflict of interest.

Publisher's Note: All claims expressed in this article are solely those of the authors and do not necessarily represent those of their affiliated organizations, or those of the publisher, the editors and the reviewers. Any product that may be evaluated in this article, or claim that may be made by its manufacturer, is not guaranteed or endorsed by the publisher.

Copyright (c) 2021 Zhou, Ying, Wu, Yang, Fu, Xu, Yao, Zeng, Huang, Lan, Wang and Liu. This is an open-access article distributed under the terms of the Creative Commons Attribution License (CC BY). The use, distribution or reproduction in other forums is permitted, provided the original author(s) and the copyright owner(s) are credited and that the original publication in this journal is cited, in accordance with accepted academic practice. No use, distribution or reproduction is permitted which does not comply with these terms. 